\title{
Las herramientas tecnológicas al servicio de gestión empresarial y administrativa.
}

The technological tools in the service of business management and administration.

Velasteguí López Efraín. ${ }^{1}$, Toaquiza V. Italo ${ }^{2}$

DOI: https://doi.org/10.33262/visionariodigital.v1i4.258

\section{Resumen.}

La presente investigación va a identificar los diversos dispositivos electrónicos y entender cuál es su impacto en las distintas operaciones que estas desarrollen en las empresas, ya que esto permitirá reconocer el funcionamiento y manejo de las herramientas dentro del ámbito laboral y su impacto para el desarrollo de su entorno.

En los últimos años se ha visto un crecimiento exponencial en el uso y la implantación de sistemas y tecnologías que mejoran la gestión de la información tanto dentro de la organización como a lo largo del canal de suministro, facilitando su difusión y la conexión entre departamentos y organizaciones. También permite simplificar las funciones rutinarias y dedicar tiempo y esfuerzo a las diferentes funciones de la organización de mayor impacto para el desarrollo de las mismas.

Adicionalmente la ya evidente globalización, juntos con la difusión de las nuevas tecnologías aplicadas al comercio electrónico han cambiado los hábitos de compra y el consumo, las estrategias de producción y las relaciones entre empresas, por estos motivos las cadenas empresariales se han visto afectadas en varios aspectos, ya que los integrantes de esas cadenas están obligadas por conseguir eficiencia, servicio y fundamentalmente por hacer rentable su entidad administrativa ya que los productores por su parte, así como los comercializadores y operadores logísticos están acogiendo rápidamente las

${ }^{1}$ Universidad Técnica de Ambato, Ambato, Ecuador, le.velastegui@uta.edu.ec 2 Toaquiza V. Italo, Ecuador 
ISSN 2602-8506

Vol. 1 / No.4/ pág. 45-61. Octubre-Diciembre/2017

www.visionariodigital.org

tecnologías más favorables para sus procesos como una forma de adecuarse y adaptarse a ese nuevo contexto competitivo dentro de la gestión empresarial y administrativa.

Palabras clave: Herramientas Tecnológicas, Empresarial, Administración, Servicios de gestión, TIC's.

\section{Introducción.}

Tecnologías de la información en logística al servicio de gestión empresarial y administrativa.

Los autores (Peralta, 2008; Goldsby, 2005; Berumen, 2007 y Garrido, 2008) establecen que las tecnologías de la información pueden ser resumidas como un conjunto de todas las actividades y soluciones producidas por una fuente computacional y con aplicaciones en muchas áreas. El termino tecnologías de la información y las comunicaciones es comúnmente utilizado para denotar el conjunto de recursos automáticos didactas al almacenamiento, procesamiento y a la comunicación de la información, así como al modo como estos recursos están organizados en un sistema capaz de ejecutar dicho conjunto de tareas.

Sabiendo que las tecnologías de la información son un elemento que ha modificado el mundo moderno en los últimos años, se determina que esta ha permitido generar procesos y operaciones hace algunos años impensables en cuanto a la combinación de la cadena suministros y a la veracidad, calidad y disponibilidad de la información.

(Arango, 2010) manifiesta que no es ajeno para ninguna persona con conocimiento en logística, que la información es un elemento fundamental en el accionar de las empresas del mundo real, en cuanto a que esta es la fuente de conocimiento de lo que ocurre en la en los escalones inferiores y superiores de la cadena de suministros, permitiendo reducir la incertidumbre asociada tanto a proveedores como a la demanda, pero fundamentalmente, que permite realmente medir, conocer y mejorar todas las instrucciones que se realicen en las organizaciones con la misma idea de la logística esbelta y de la administración de la cadena de suministro: Optimizar los procesos y las operaciones.

Las tecnologías de la información y las comunicaciones son una fuente para la estimulación de la innovación en los servicios, incrementar la eficiencia en la producción y al mismo tiempo facilitar los procesos de manejo de inventario y la administración de costos. Estas tecnologías han favorecido la organización dentro de las empresas y han permitido reducir los costos de operación de las actividades propias de la empresa y de aquellas asociadas a la cadena de suministro.

Uno de las principales características de las tecnologías de la información asociada a las empresas es que estos beneficios tienen un impacto a largo plazo en empresas nuevas como en aquellas tradicionales. Por otro lado las tecnologías de información y las comunicaciones son considerablemente importantes en el desarrollo y éxito de las compañías, ya que permiten determinar las características de las compañías que conllevan 
a obtener ventaja competitiva, elemento fundamental en el mundo actual, ya que cada vez es más difícil de identificar debido a los frecuentes y rápidos cambios del mercado, así como a la incertidumbre de los ambientes de negocio.

Determina (Arango, 2010) que la importancia de las tecnologías de la información en la logística y en la administración de la cadena de suministros radica en que éstas hacen posible lo que la información correcta esté disponible en el momento y el lugar adecuado cuando se requiere, lo cual permite que el flujo de inventario a través de la empresa y de la cadena de suministro se comporte de forma similar a como lo hace la información.

De esta forma, muchas compañías están generando soluciones tecnológicas para mejorar los elementos y tareas mal realizadas dentro de sus operaciones y para facilitar la administración de las mismas. Mientras que las tecnologías de la información son continuamente efectivas en la captura y procesamiento de información que sirve como medida del desempeño de una operación o una persona, estas también presentan la característica de permitir al sistema el mantenimiento del mismo. La información dentro de una empresa fluye a través de la compañía en diferentes formas, a diferente velocidad y por muchos motivos.

(Arango, 2010) menciona que la información es usada para administrar el presente, planificar el futuro y reflejar el pasado. Administrar toda la información puede ser una tarea desalentadora e insoportable, pero es una actividad que debe hacerse y desarrollar exitosamente para el correcto funcionamiento de las organizaciones.

\section{Tecnologías de la información como flujos de información.}

Según (Goldsby, 2005 y Taylor, 2008) determinan que existen tres áreas más relevantes y estratégicas sobre las cuales se encuentran los flujos de información.

- Flujo de datos.

- Flujo de conocimiento.

- Flujo de comunicación.

En las tres áreas de las acciones logísticas se determina que estas generan más información que cualquier otro oficio empresarial, razón por la cual es función de los profesionales en logística buscar la mejor forma de utilizar la información disponible.

(Arango, 2010) determina que la idea central es convertir los datos en información y la información en conocimiento. Sin embargo, no todos los datos producen información, es necesario que el personal de logística verifique los datos puede convertir en información dependiendo de las necesidades de los mismos. Una de las ventajas más importantes al obtener eficiencias en la adquisición, manejó e intercambio de información a través de la cadena de suministro, es que mediante esta se puede eliminar las variaciones en la percepción erróneamente exagerada de la demanda. 
Lo anterior es apenas lógico al entender que entre mayor sea el flujo de datos e información que se tenga sobre cada elemento que compone la cadena de suministros, más fácil y precisa se hacen las predicciones de las necesidades futuras.

Para (Arango, 2010) los procesos de gestión y análisis de decisiones que los administradores de dicha cadena de suministros, deben complementar sobre algunos aspectos, se deben enfocar claramente en los tres principales grupos de procesos de la cadena antes mencionada, e ir denominados procesos macro, dando a la necesidad de obtener un enfoque holístico, pues el éxito de la misma depende del resultado global y no del particular en algunas de las operaciones internas, puesto que alguno de los procesos los cuales son la administración de las relaciones con clientes o la cadena de suministros interna y la administración de las relaciones con los proveedores, todos estos aspectos deben estar soportados o desarrollados sobre las tecnologías de la información que se ofrecen en cuanto a que en cada una de ellos fundamentalmente tenga una integración propia.

Teniendo en cuenta que la gestión de los sistemas de información, se deben enfocar independientemente en cada uno de los diferentes procesos Macro y fundamentalmente en la adecuada portada diseñada para la administración del proceso de funcionamiento de la administración de las transacciones cabe mencionar que a primera vista la importancia de las transacciones de donde saltan a primera vista la importancia de la correcta selección y administración de las tecnologías de la información asociadas a la logística que como bien sabemos las tecnologías de la información habilita varios procesos Macro así como su integración de los sistemas efectivos de integración no sólo permiten obtener los datos de toda la cadena de suministro sino también de análisis de las decisiones que maximizan la rentabilidad de la misma.

\section{Captura y transferencia de la información con las herramientas tecnológicas}

Manifiestan (Arango, 2010 y Fernandez, 2004) en sus estudios que la transferencia de la información juega un papel fundamental dentro de la logística, Pues de la velocidad de esta depende la correcta operación de las actividades logísticas, así como la reducción del efecto látigo en las organizaciones. Las herramientas para la captura y transferencia de la información, más relevantes en logística son el internet la banda ancha el Eddy y el xml.

Determina (Arango, 2010) que dentro de las herramientas que esta ofrece tenemos el internet ya que esta herramienta es la red de comunicación de datos globales que utiliza el formato y para compartir la información desde un lugar a otro. El internet es luego una red de comunicación universal a la que puede conectarse cualquier computador y cualquier dispositivo. Una de las principales características del Internet, es que al conectarse es posible comunicarse con cualquier otro dispositivo que igualmente esté conectado a la Red, sin importar los diferentes formatos de información que estos dispositivos utilicen. Con el internet múltiples compañías pueden conectarse subsistemas de computadores y operar entre ellos de una forma rápida y casi sin costo. El internet permite trabajar con una protección extra de la información. 
De esta manera el internet en si es un conjunto descentralizado de redes de comunicación interconectadas que lo utilizan la familia de protocolos TCP/IP y son de alcance mundial, a su vez ayudan con la comunicación y al desarrollo laboral y globalizado. De la misma manera tenemos lo que es la banda ancha que es básicamente cualquier tecnología de comunicaciones que facilita acceder a internet a altas velocidades. La banda ancha permite a las empresas conectarse internamente utilizando tecnología de redes de área local LAN como es el caso de la Ethernet, la cual ofrece alta capacidad de comunicación interna. Por otro lado, la comunicación con las compañías o plantas en diferentes posiciones geográficas, es posible mediante la tecnología de redes de área amplia WAN.

De esta forma, las principales ventajas de la banda ancha es la alta velocidad de transferencia de la información, relativo bajo costo y con gran capacidad de conectividad interna y externa.

Menciona (Arango, 2010) que otro de los aspectos son el Intercambio Electrónico de Datos (EDI) que básicamente son el intercambio electrónico de datos es una tecnología desarrollada para enviar datos mediante tipos de comunicación comunes entre las distintas compañías. Las herramientas involucran la transmisión electrónica de información y documentos, como por ejemplo: facturas u órdenes de compras directamente entre computadoras de diferentes empresas basados en formatos estándares, estructurados y mecanizables. Los estándares del EDI llamados X.12, son fijados por el Instituto Nacional americano de Astándares (ANSI) y son especializados entre industrias.

Determinando que mediante la estandarización del intercambio de documentos, el EDI permite la eliminación de ingreso de la información y los errores asociados a la interacción humana.

Esta herramienta también permite la reducción de costos, incremento en la productividad, disminución del uso del papel al mínimo y mejora las relaciones entre compañías.

\section{Almacenamiento y a la recuperación de la información en las empresas}

Para (Frezelle, 2007 y Bruque, 2003) las tecnologías de la información destinadas al almacenamiento y a la recuperación de la información son las bases de datos, las cuales se conciben como la organización de los datos y la información que es guardada electrónica mente.

Se determina que existen varios tipos de bases de datos, dónde la más común es la base de datos relacional, la cual almacena grupos de datos relacionados en tablas individuales que permiten la recuperación mediante el uso de un lenguaje estándar llamado sql_ structured query Language.

Según (Arango, 2010) manifiesta que una base de datos estará definida por el nivel de detalle de la información que se almacena, dicho eso la variable más importante vendría a ser el balance entre tener una información altamente agregada o altamente detallada de lo que se vaya almacenar, lo cual depende de las necesidades y especificaciones de los 
ISSN 2602-8506

Vol. 1 / No.4/ pág. 45-61. Octubre-Diciembre/2017

www.visionariodigital.org

usuarios y esto también vendría a tener un costo al momento de almacenar la información altamente detallada.

Cabe mencionar que la característica fundamental de una base de datos. De las bases de datos es que permite que las transacciones puedan ser registradas almacenadas y posteriormente consultar y es el mismo de la base de datos quién determina qué operaciones realizará, en qué nivel de detalle, con qué secciones realizar a dichas transacciones, estas son registradas y vigiladas además de estas transacciones son registradas pueden ser observadas en tiempo real o por baches del tiempo lo cual es determinado por el administrador del sistema

(Arango, 2010) menciona que también permiten entregar la información almacenada de diferentes formas, dependiendo de las necesidades de los usuarios, éstas diferentes formas de ver la información se conocen con el nombre de vistas y se deben a que no todos los usuarios pueden o necesitan ver la totalidad de información contenida en una búsqueda específica.

Por ejemplo se asume que cuando el personal de ventas de una compañía está interesado en obtener información sobre un producto, este seguramente está buscando información relacionada con históricos de venta, o también precios de fabricación, de producción, garantías, entre otros, mientras que una persona de producción no estará, ni tendrá por qué estar interesada en esta información y por el contrario él buscará datos concernientes a materias primas, condiciones de operación, entre otros parámetros.

\section{Identificación y entrega de información con las herramientas tecnológicas}

Los autores (Berumen, 2007; Chopra, 2008; Fernandez, 2004; Lethan, 2002 y LRM, 2018) definen a estas tecnologías asociadas como la coordinación sistemática y estratégica de las funciones de negocios tradicionales y tácticas utilizadas a través de estas ya sea al interior de una empresa y entre los diferentes procesos de una cadena de suministro con el fin de mejorar el desempeño en un largo plazo tanto de las empresas o de toda una cadena de suministros.

Para las cuales se determinan las más importantes como son el código de barras que esta es un sistema de codificación de productos que de forma inequívoca identifica la mercancía. Estos códigos permiten dicha identificación de forma rápida y sin papeleo, lo que conlleva a una operación del proceso de entrega y despacho más eficiente. De esta manera se disminuyen los costos asociados al tiempo de identificación y a los costos de errores de enviar y recibir productos erróneos.

Mencina (Arango, 2010) que para adherir la etiqueta al producto los códigos de barras se pueden utilizar para: identificación de productos.

- Identificación de contenedores

- Identificación de ubicaciones 
- Identificación de operadores

- Identificación de equipos

- Identificación de documentos

También los códigos están diseñados para alto volumen de lectura, motivo por el cual son muy utilizados en las tiendas de grandes superficies. El código de barras se identifica con los primeros 3 dígitos que corresponden al código del país de fabricación, del 4 al séptimo dígito identifican la compañía productora, los dígitos 8 a 12 dan la información referente al tipo de producto y el último dígito es dígito de verificación.

Para (Arango, 2010) otro importante elemento es la radiofrecuencia que esta es una tecnología de uso reciente en almacenamiento e identificación de productos y SKU. Esta trata de conectar por radio, sin cables, un elemento de manipulación, la computadora central. De esta forma, se ahorran tiempos de desplazamiento las ubicaciones y el stock se actualizan en línea automáticamente. Básicamente se determina que existen dos tipos de TAGS de radiofrecuencia, los pasivos y los activos, los primeros no tienen fuente de alimentación independiente y pueden tener un alcance entre $2 \mathrm{~cm}$ y 13 metros, los activos que poseen una batería o fuente propia pueden ser leídos desde una distancia de varios kilómetros.

Las bandas magnéticas y tarjetas ópticas son un elemento importante también ya que se usan para almacenar gran cantidad de información en un espacio pequeño y se pueden leer aún en condiciones de poca limpieza. Las bandas magnéticas y tarjetas óptica se utilizan para diversas funciones, que van desde la identificación de empleados hasta la composición de carga de material en un camión o la composición de un recorrido para preparación de un pedido.

(Arango, 2010) determina que tenemos al GPS que es el sistema de posicionamiento global por sus siglas en inglés, es un sistema de satélites, que permiten durante las 24 horas del día, conocer la posición de un dispositivo en la superficie terrestre. El sistema de posicionamiento global se determina que consiste en un conjunto de 24 satélites que circundan la tierra y envían señales de radio a la superficie. Para esto se necesita un receptor GPS que es un dispositivo electrónico pequeño utilizado por aquellos que viajan por la tierra mar y aire que permite recibir las señales de los satélites. Este receptor utiliza las señales de radio para calcular la posición que es entregada como un grupo de número y letras correspondientes a un punto sobre un mapa.

Para la sincronización de las entregas y la administración de flotas de carga las compañías utilizan asistencia de posicionamiento satelital, con la cual pueden conocer en todo momento en qué lugar se encuentran los vehículos, mejorando notablemente el sistema de entrega y distribución mediante la trazabilidad, seguimiento y auditoría de los envíos.

Para (Arango, 2010) otro elemento importante a detallarse seria la Selección de voces que prácticamente es la estructura básica del sistema Voicepicking. Los sistemas de VOICEPICKING o Selección de voces son los sistemas en los cuales el operario recibe 
las instrucciones del ordenador mediante un sistema de recepción que convierte la señal en una instrucción verbal, la cual es escuchada por el operario a través de un auricular.

Determinando que de forma similar, igual puede retroalimentar al sistema mediante un micrófono y el receptor, el cual transmite la información convirtiéndola en una señal capaz de ser procesada por el ordenador central. De esta forma, el operario continuamente está hablando con la computadora central, la cual le está dando las órdenes de que materiales y la cantidad a recoger en posición del almacén.

(Arango, 2010) determina que el Pick to Light que es un sistema que ayuda al operario en la recolección de mercancías mediante una secuencia de ayudas visuales que permiten que esté llegué directamente a la posición deseada. Para su identificación, cada posición dentro del almacén posee un dispositivo con una pantalla digital, la cual indica al recolector la cantidad de elementos a recoger, e inclusive permite realizar operaciones de chequeo, corrección o modificación de las órdenes.

Este dispositivo se conoce como digital DPDs (pinking display) el cual se esquematiza en la siguiente figura.

Nombre de imagen: DPDs de un sistema de Picking to Ligth

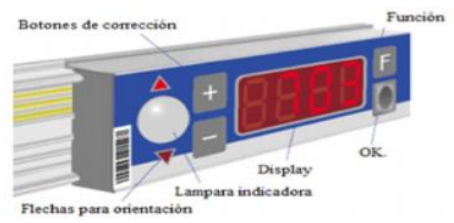

Fuente y Autor: Exomexico

El siguiente elemento es se mucha importancia ya que ayuda al reconocimiento visual automático tiene una precisión mayor que los sistemas voz y esta rata de ser una solución $100 \%$ libre de errores, esta herramienta acelera los tiempos de entrenamiento de personal nuevo y al igual que en ambas manos quedan libres para realizar el proceso recolección.

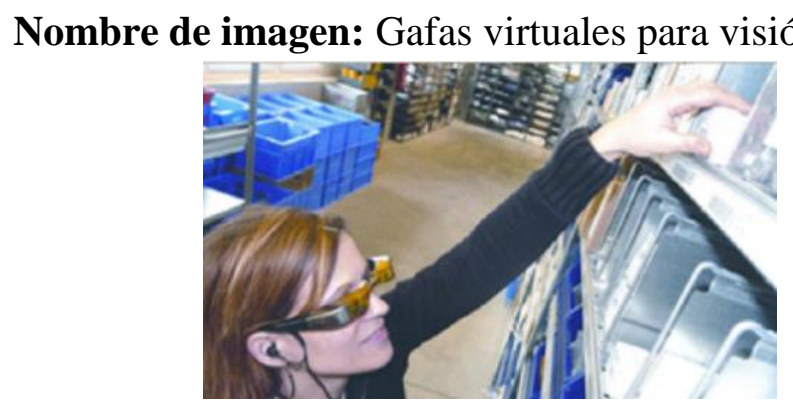

Fuente: freelogistics.com

\section{Administración y reporte de la información con las herramientas tecnológicas}

Para los autores (Taylor, 2008; Canul, 2018 y Lira, 2018) mencionan que el ERP y los sistemas de ejecución de la compañía, son los componentes más importantes de los sistemas de la información en logística, ya que proveen bases de datos de transacción para 
inicializar, medir, monitorear y reportar acerca de órdenes provenientes de los clientes o hacia los proveedores, los sistemas de ejecución empresariales proveen la interfaz entre el ERP y las operaciones diarias con los clientes transportadores y el almacenamiento a través de los sistemas, el beneficio final de un ERP es alcanzar la integración entre la compañía, los proveedores y los clientes.

El sistema de planificación de requerimientos de materiales son un paquete de programas de gestión de los flujos de administración de la empresa a su vez de un sistema de información donde los diferentes tratamientos tanto transacciones y ejecución y las distintas funciones clásicas de una empresa son integradas alrededor de las bases de datos y de un herramienta de procesamiento que son de gestión integrados para las empresas soportados por un paquete de programas integrados y parametrizables de éstos los cuales permiten administrar el conjunto de las funciones y los procesos de la entidad.

(Arango, 2010) Menciona que las empresas de los ERP están divididas en tres grandes grupos de proveedores: SAP, las empresas que adquiridoras y las empresas independientes. SAP es una empresa alemana que ofrece un SAP bajo su nombre y la cual lidera el mercado de los SAP a nivel mundial. Se dice también que los adquiridores incluyen vendedores como Oracol, Microsoft Business Solutions, SSA Global e Inffor, todos los cuales han crecido a través de adquisiciones de otras compañías. Los independientes son por el contrario las casas de software independiente y en algunos casos de software libre tales como intenta, IFS, IBS, QAD Y Epicor. Los sistemas de abastecimiento Procurement System vienen siendo son sistemas especializados en las actividades de compras realizadas entre la compañía y los proveedores.

El objetivo fundamental de estos sistemas es dinamizar y hacer más eficientes las operaciones de abastecimiento. Se determina que existe una nueva generación de sistemas ERP están evolucionando para producir interacciones adicionales, especialmente para clientes o con clientes, estos sistemas identificados como ERP 2, integran el ERP tradicional como un sistema manejador de relaciones de clientes para integrar mejor los requerimientos de los clientes, las claves con las plantas de la compañía.

Para (Arango, 2010) la mejora ofrecida por esta compañía es la conectividad externa lo cual es crucial para la colaboración a lo largo de la cadena de suministro esta herramienta adicionalmente es la posibilidad de integrarse con internet con lo cual se permitiría el mando del sistema desde cualquier lugar del planeta bastando sólo una conexión a internet apropiada.

Generalmente estas vendrían a reemplazan los catálogos por bases de datos que contienen toda la información requerida acerca de los productores que la compañía requiere, al tiempo que también evalúa el número de unidades compradas, precio de compra, historial de compras y desempeño de proveedores.

\section{Evolución en gestión de la empresa con las tecnologías de la información}

Según los autores (Canul, 2018 y Arango, 2010) definen a la empresa como en una unidad económico-social integrada por elementos humanos, materiales y técnicos que 
tiene el objetivo de obtener utilidades a través de su participación en el mercado de bienes y servicios. Para esto se hace uso de los factores productivos (trabajo tierra y capital).

La gestión empresarial y administrativa viene siendo aquella actividad empresarial que a través de diferentes individuos especializados como: directores institucionales, consultores, productores gerentes, entre otros, y acciones buscará mejorar la productividad y la competitividad de una empresa o de un negocio.

Determina (Arango, 2010) que la gestión empresarial hace referencia a las medidas y estrategias llevar a cabo con la finalidad de que la empresa sea viable económicamente. Es una de las principales virtudes de un hombre de negocios engloba las distintas competencias que se deben tener para cubrir distintos flancos que de una determinada actividad comercial en el contexto de una economía de mercado.

La dirección de la empresa en base al concepto de la gestión implica un elevado nivel de comunicación por parte de los administradores para con los empleados y esto nace a partir de tener el objetivo de crear un ambiente adecuado de trabajo y así aumentar la eficiencia del trabajo de los empleados aumentando las responsabilidades de la empresa. El control es la función final que debe cumplir el concepto de gestión aplicado a la administración, ya sea de este modo se podrá cuantificar el progreso que ha demostrado el personal empleado en cuanto a los objetivos que se les habían sido marcados desde un principio.

Para (Arango, 2010) la gestión hace referencia a la acción y a la consecuencia de administrar o gestionar algo. Administrar Por otra parte abarca las ideas de gobernar disponer dirigir ordenar y organizar una determinada cosa o situación. Gestiones es la asunción y ejercicio de responsabilidades sobre el proceso, es decir sobre un conjunto de actividades, lo que incluye:

- La preocupación por la disposición de los recursos y estructuras necesarias para que tengan lugar.

- La coordinación de sus actividades y correspondientes interacciones

- La rendición de cuentas ante el abanico de agentes interesados por los defectos que se espera que el proceso desencadene.

(Arango, 2010) manifiesta que las funciones de gestión de las existencias permiten contabilizar las entradas y las salidas producidas y así controlar el estado de las existencias en cualquier momento, en la gestión de las existencias permiten también caminar la mercancía recibida hacia el emplazamiento adecuado está a su vez tiene una funcionalidad también que permite poner en marcha las órdenes de reabastecimiento según los led Times, cantidades en existencia y los umbrales que existen por definirlos.

De acuerdo con la arquitectura empresarial se determinan que es una herramienta de tecnología para el manejo y gestión de una organización, la cual adopta una vista integral que cubre desde sus procesos de negocio, los sistemas de información, los datos de información y la infraestructura tecnológica, permitiendo explicar como todos los elementos de las tecnologías de la información es una organización, los procesos, los 
ISSN 2602-8506

Vol. 1 / No.4/ pág. 45-61. Octubre-Diciembre/2017

www.visionariodigital.org

sistemas, la estructura organizacional y las personas se integran y trabajan de forma conjunta.

\section{Dispositivos utilizados para el desarrollo de la gestión empresarial}

Las empresas tienen cada vez más opciones para decidir con qué dispositivos informáticos equipar a sus empleados, por lo que también aumenta sus dudas sobre el cuál es el más adecuado.

(Fernandez, 2004 y Canul, 2018) establecen en sus investigaciones que por muy buenas que sean las pantallas táctiles en muchos casos se necesita un teclado y un ratón, por lo que la mayoría de las compañías utilizan un PC o un MAC", analista de Gartner Inc., consultora de tecnologías de la información.

Aunque los aparatos móviles, como tabletas y los Smartphone se han extendido durante los últimos años, los ordenadores de sobremesa y las portátiles todavía mantienen liderazgo.

Las tabletas útiles para viajar ya que a los trabajadores que tienen que viajar utilizan cada vez más los programas de almacenamiento en la nube, lo que a su vez favorece la extensión de las tabletas, al tener que almacenar archivos e información, los dispositivos móviles se convierten en una opción más cómoda y útil para los desplazamientos.

También existen algunos de los dispositivos móviles de manufacturación y pedidos La unión entre movilidad y Software profesional es una realidad mezclar durante los últimos años la evolución del sector Smartphone ha tenido un gran impacto tanto a nivel de consumo general como a nivel profesional y esto ha repercutido en las herramientas de trabajo que utiliza empresas y autónomos.

A continuación se detallaran algunos de los productos competitivos de dispositivos de recolección de datos proveedores y dispositivos de recolección de datos fabricantes. Tenemos el dispositivo de la compañía Rakinda, su Función es que el equipo de pedido de almacén, recolectara de datos mano/móvil, PDA móvil de código de barras y colector de datos.

Cuenta con un procesador de frecuencia dominante1,2 con una capacidad de memoria RAM de 2G, ROM de $16 \mathrm{G}$ y con un sistema operativo Android 5,1 estilo PDA scanner y muchos de los dispositivo de recolección de datos y pedidos tienen las mismas características de funcionamiento.

Nombre de imagen: Dispositivo Rakinda

RAKINDA

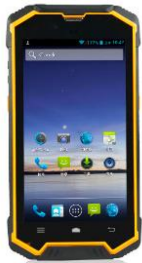


ISSN 2602-8506

Vol. 1 / No.4/ pág. 45-61. Octubre-Diciembre/2017

Fuente y Autor: Alibaba.com

\section{Uso de las herramientas tecnológicas, panorama empresarial en el Ecuador y América del Sur}

Para (Delgado, 2015) los indicadores del banco mundial muestran que entre 2005 y 2012 se constató un incremento significativo del número de empresas en América del sur, donde Perú y Chile son los países que presentaron mayor crecimiento de la densidad empresarial, dejando al resto de países como Bolivia y Surinam y, extrañamente a la Argentina entre los últimos lugares.

De la información encontrada se determina que el crecimiento en cuanto al uso de las tecnologías de la información han sido un elemento muy importante en el desarrollo empresarial dentro de América del Sur, haciendo de algunos de estos países que los utilizan, más efectivos en cuanto al entorno laboral.

Grafico 1. Densidad de las nuevas empresas en países seleccionados de América del Sur (2005-2012). Registros nuevos por cada 1000 personas

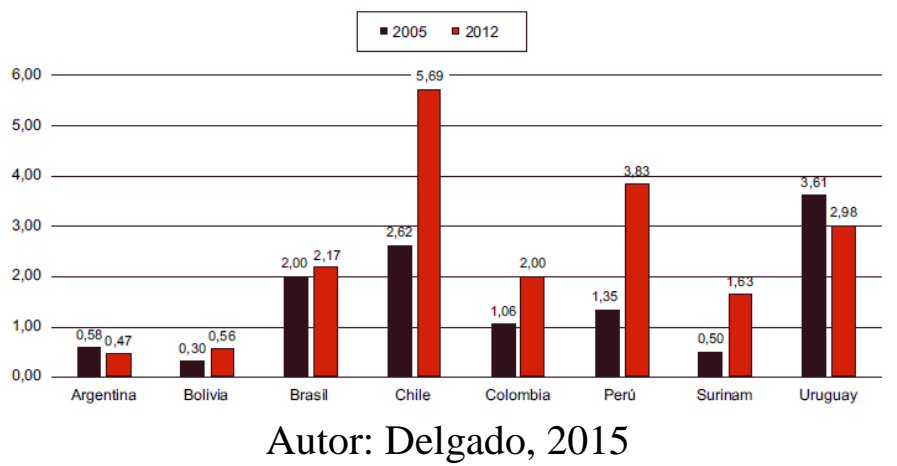

Teniendo en cuenta que el nivel de simplicidades que se retracta América del sur ha ocasionado que las diferentes entidades empresariales cuenten con un porcentaje menos al acceso al crédito, esto a su vez hace que esto sea menos productivo en su actividad.

Se a constatado que según los indicadores del Banco mundial, se verifica que ha existido en el año 2012 una informalidad muy elevada en la región, sabiendo de esto que el $70 \%$ de las entidades empresariales han empezado la actividad de forma ilegal, lo que da lugar a que las empresas no puedan empezar netamente establecidas las actividades

Grafico 2: Empresas formalmente establecidas al inicio de sus operaciones en América del sur. 


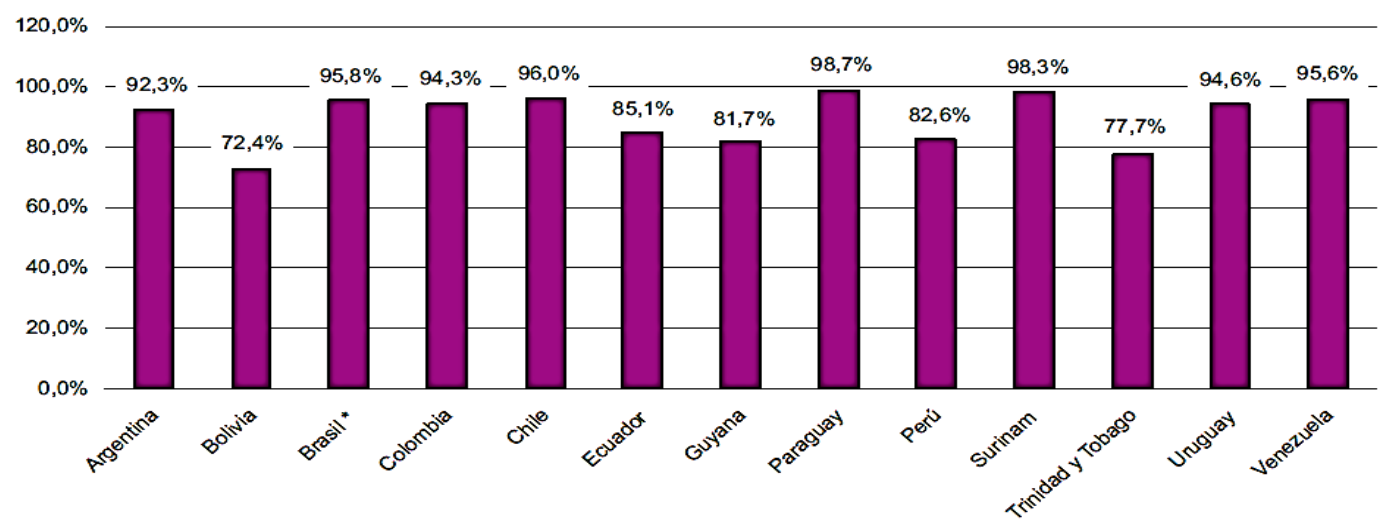

Autor: Delgado, 2015

También hay que tener en cuenta la importancia de las tecnologías en la organización de cada una de las entidades, lo cual hace de suma importancia ver de una manera generalizada el flujo de informaciones se va a administrar y como se lo va a ejecutar, para eso tenemos una gráfica en donde nos indicara algunos aspectos de información de uso y su disponibilidad y el uso del mismo.

Cuadro 1. Áreas de uso de las herramientas tecnológicas

\begin{tabular}{|c|c|c|}
\hline $\begin{array}{l}\text { Articulación } \\
\text { de } \\
\text { la empresa } \\
\text { con: }\end{array}$ & $\begin{array}{l}\text { Tareas } \\
\text { digitalizables: }\end{array}$ & Beneficios: \\
\hline Clientes & $\begin{array}{l}\text { Gestión de pedidos } \\
\text { Facturación } \\
\text { Control de clientes } \\
\text { Historial de pedidos } \\
\text { Información sobre } \\
\text { productos disponibles } \\
\text { Localización de } \\
\text { clientes potenciales }\end{array}$ & $\begin{array}{l}\text { Más canales de comunicación } \\
\text { Menores costos de transacción } \\
\text { Ampliar horario de atención } \\
\text { Mayor gestión } \\
\text { Reducción de costos de factoraje } \\
\text { Compartir información } \\
\text { Interacción constante } \\
\text { Estado de las operaciones en tiempo real } \\
\text { Mejor gestión de inventarios }\end{array}$ \\
\hline $\begin{array}{l}\text { Entre áreas o } \\
\text { departamentos }\end{array}$ & $\begin{array}{l}\text { Seguimiento del ciclo } \\
\text { de producción } \\
\text { Área de diseño } \\
\text { Planeación de } \\
\text { producción } \\
\text { Control de inventarios } \\
\text { Gestión de } \\
\text { mantenimiento de } \\
\text { maquinaria y equipo } \\
\text { Reportes del personal }\end{array}$ & $\begin{array}{l}\text { Seguimiento del ciclo de producción } \\
\text { Área de diseño } \\
\text { Planeación de producción } \\
\text { Control de inventarios } \\
\text { Gestión de mantenimiento de maquinaria } \\
\text { y equipo } \\
\text { Reportes del personal } \\
\text { Control de calidad } \\
\text { Contabilidad } \\
\text { Nómina }\end{array}$ \\
\hline
\end{tabular}


ISSN 2602-8506

WWw.visionariodigital.org

\begin{tabular}{|c|c|c|}
\hline & $\begin{array}{l}\text { Control de calidad } \\
\text { Contabilidad } \\
\text { Nómina }\end{array}$ & \\
\hline Proveedores & $\begin{array}{l}\text { Gestión de pedidos } \\
\text { Facturación } \\
\text { Control de } \\
\text { proveedores } \\
\text { Historial de pedidos } \\
\text { Información sobre } \\
\text { productos disponibles } \\
\text { Localización de } \\
\text { nuevos proveedores }\end{array}$ & $\begin{array}{l}\text { Más canales de comunicación } \\
\text { Menores costos de transacción } \\
\text { Horario de atención } \\
\text { Mayor gestión } \\
\text { Reducción de costos de factoraje } \\
\text { Compartir información } \\
\text { Interacción constante } \\
\text { Estado de las operaciones en tiempo real } \\
\text { Mejor gestión de inventarios }\end{array}$ \\
\hline
\end{tabular}

Autor: Casalet y Gonzalez(2004)

Por otro lado los países de Brasil y chile se determinan como uno de los países que presentan una disponibilidad de tecnologías operables, lo que hace de estos lugares pioneros en el uso de las tecnologías en este sector empresarial de cualquier índole haciendo de esto algo netamente innovador.

En el siguiente grafico veremos el porcentaje de páginas web que utilizan las distintas empresas en los diferentes países.

Grafico 3. Páginas web utilizadas para promocionar las empresas en los diferentes países.

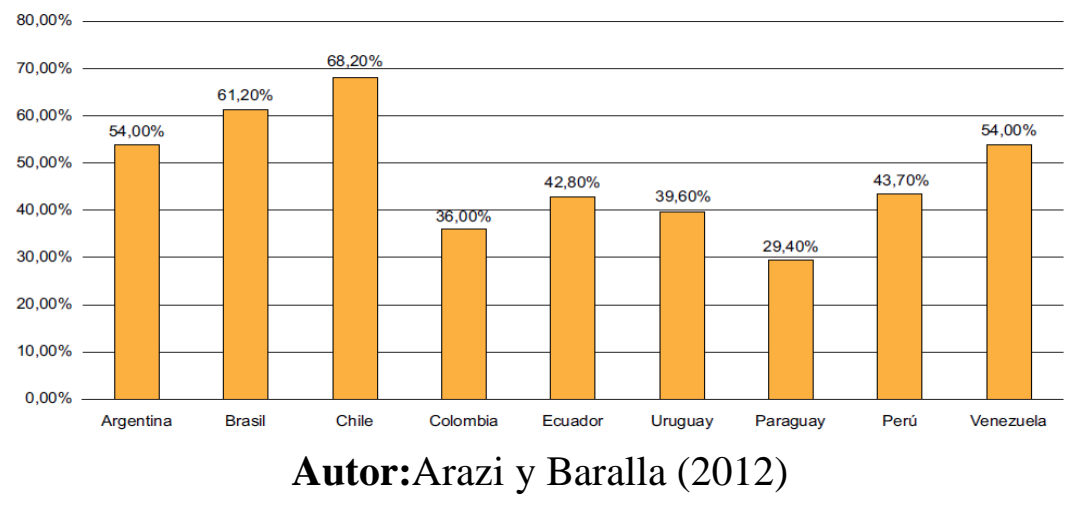

\section{Conclusiones:}

- Se determina mediante lo anteriormente expuesto que los dispositivos electrónicos y las tecnologías de la información son herramientas que permiten mejorar las distintas actividades que se desarrollan en las distintas empresas, las cuales pueden ser herramientas para fines operativos, como recolección de información, pueden ser destinadas a fines administrativos como los sistemas de administración de inventarios, entre otros. 
- Para estas situaciones existe un gran número de herramientas tecnológicas disponibles en el mercado, las cuales deben ser tenidas en cuenta y profundamente analizadas por una correcta selección de la tecnología a utilizar.

- Se determina una caracterización de las tecnologías de la información asociadas a la gestión empresarial y administrativa, constatando los distintos aspectos como son las tecnologías asociadas a la captura y transferencia de la información, herramientas destinadas al almacenamiento y recuperación de la información, tecnologías con fines de identificación y entrega de información y por último también las tecnologías utilizadas para la manipulación, administración y reporte de la información lo cual caracteriza esta forma con el propósito de generar una concepción amplia de las tecnologías en la administración y gestión empresarial.

- El uso de las herramientas existentes en el medio tecnológico son y será un aspecto de desarrollo tanto económico, social, y laboral, haciendo de este proceso común a un proceso de mayor eficiencia al momento de desarrollar las actividades laborales dentro de una entidad administrativa y laboral.

\section{Bibliografía:}

Arango, M., Londono, E., \& Zapata, J. (2010). Arquitectura Empresarial- Una vision general (16 ed.). Medellin: Universidad de medellin.

Arazi, M., \& Baralla, G. (2012). www10.iadb.org. Recuperado el 31 de Noviembre de 2018, de http://www10.iadb.org/intal/intalcdi/PE/2013/

Berumen, S. (2007). Cambio tecnologico e innovacion en las empresas. Medellin: Esic Editorial.

Bruque, S., Vargas, A., \& Hermnandez, M. (2003). Determinantes del valor competitivo de las tecnologias de la informacion. Una aplicacion al sector de la distribucion farmaceutica. Madrid: Revista Europea.

Canul Canul, D. (20 de octubre de 2018). slideshare.net. Obtenido de https://es.slideshare.net/Ofimaticos/gestin-empresarial-y-10-herramientastecnologicas-para-la-gestin-empresarial

Casalet, M., \& González, L. (2004). Las tecnologías de la información en las pequeñas y medianas empresas mexicanas. Acapulco: Revista electrónica de geografía y ciencias sociales.

Chopra, S., \& Meindl, p. (2008). Administracion de la cadena de suministro. Estrategia, planificacion y operacion. Mexico: Pearson Education.

Delgado Aranda, M., \& Simão de Paula , P. (2015). Empresa y tecnologías de la información: una mirada a Bolivia y América del Sur (Vol. 10). Buenos Aires, Argentina: Revista Iberoamericana de Ciencia, Tecnología y Sociedad - CTS. 
WwW.visionariodigital.oro

Fernandez, G. (2004). Conocimientos y aplicaciones tecnológicas para la dirección comercial. Madrid: Esic Editorial.

Frezelle, E., \& Sojo, R. (2007). Logistica de Almacenamiento de Clase Mundial. Bogota: Grupo editorial Noma.

Garrido, a., Ferreira, J., \& Leitao, J. (2008). The role of logistics information and communication technologies in promoting comparative advaantages of firm. (Vol. 5). Mexico: The Icfai.

Goldsby, T., \& Martichenko, R. (2005). Learn six Sigma Logistic. Strategic Develoment to operational success. San Antonio: J. RossPublishing, INC.

GS1 Colomba. (2018). Estandar GS1 Código de Barras. ASPX. Obtenido de http: //www.gslco.org

Lethan, L. (2002). Uso del sistema de posicionamiento Global. Paidotribo Editorial, 1, 50-65.

Lira Segura, J. (20 de Octubre de 2018). gestion.pe. Obtenido de https://gestion.pe/tendencias/management-empleo/son-dispositivos-utilizadospymes-145794

LRM, c. (20 de Junio de 2018). Irmconsultoriaalogistica.es.blog. Obtenido de www.Irmconsultoriaalogistica.es/blog/feed/9-articulos/32-vision-picking.html

Marimo, S. (1999). La sanidad en la sociedad de la informacion: sistemas y tecnologias de la informacion para la gestion y la reforma de los servicios de salud. Caracas: Ediciones Diaz de Santos.

Peralta, F., \& Vieira, J. (2008). Information Technology and Communication and Best Practices In It Lifecycle Management, J. Technol. Manag. Innov (Vol. 3). Orlando: Issue 4.

Rosettanet. (20 de octubre de 2010). Rosettanet.org. Obtenido de www.rosettanet.org Taylor, G. (2008). Logistics engineering handbook. USA: CRC press.

Vargas, A., Bruque, S., \& Hernandez, M. (2003). Determinantes del valor competitivo de las tecnologias de la informacion (Vol. 12). Madrid: Revista Europea.

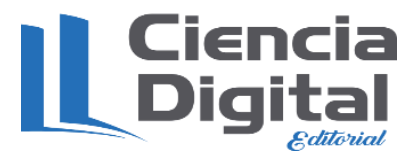




\section{Para citar el artículo indexado.}

Velasteguí E. , Toaquiza I. (2017). Las herramientas tecnológicas al servicio de gestión empresarial y administrativa. Revista electrónica Visionario Digital 1(1), 45-61. Recuperado desde: http://cienciadigital.org/revistacienciadigital2/index.php/VisionarioDigital/article/view/ $258 / 561$

\section{【Liencia}

El artículo que se publica es de exclusiva responsabilidad de los autores y no necesariamente reflejan el pensamiento de la Revista Ciencia Digital.

$\mathrm{El}$ articulo queda en propiedad de la revista y, por tanto, su publicación parcial y/o total en otro medio tiene que ser autorizado por el director de la Revista Ciencia Digital. 